\title{
AN EXTENSION OF A THEOREM OF HARTOGS
}

\author{
BY
}

L. R. HUNT

\begin{abstract}
Hartogs proved that every function which is holomorphic on the boundary of the unit ball in $C^{n}, n>1$, can be extended to a function holomorphic on the ball itself. It is conjectured that a real $k$-dimensional $\mathscr{C}^{\infty}$ compact submanifold of $C^{n}, k>n$, is extendible over a manifold of real dimension $(k+1)$. This is known for hypersurfaces (i.e., $k=2 n-1$ ) and submanifolds of real codimension 2 . It is the purpose of this paper to prove this conjecture and to show that we actually get $\mathrm{C}-\mathrm{R}$ extendibility.
\end{abstract}

1. Introduction. Let $M^{k}$ be a real $k$-dimensional compact $\mathscr{C}^{\infty}$ manifold embedded in $C^{n}, k, n \geqq 2$. Hartogs proved that every function holomorphic in an open neighborhood of $M^{2 n-1}$ can be extended to a function holomorphic in some open subset of $\boldsymbol{C}^{n}$. Bochner proved a similar theorem for functions which satisfy the induced Cauchy-Riemann equations on $M^{2 n-1}$. It has been conjectured that any real $k$-dimensional compact $\mathscr{C}^{\infty}$ submanifold of $\boldsymbol{C}^{n}$ is extendible to a manifold of real dimension $(k+1)$ if $k>n$. This has been proved for real-analytic submanifolds of $C^{n}$ in [3] and generic C-R submanifolds in [2]. It is the purpose of this paper to prove the conjecture with extendibility being replaced by $C-R$ extendibility.

The early work for the higher codimensional study was done by Bishop [1], Wells [6] and Greenfield [2]. A recent article due to Nirenberg [4] led to the results in this paper.

2. Definitions. Let $M^{k}$ be a real $k$-dimensional $\mathscr{C}^{\infty}$ manifold embedded in $\boldsymbol{C}^{n}$, $k, n \geqq 2$. Suppose $T\left(M^{k}\right)$ is the tangent bundle to $M^{k}$, and $J$ denotes the almost complex tensor $J: T\left(C^{n}\right) \rightarrow T\left(C^{n}\right)$, with $J^{2}=-I$. Then we define

$$
H_{p}\left(M^{k}\right)=T_{p}\left(M^{k}\right) \cap J T_{p}\left(M^{k}\right),
$$

the vector space of holomorphic tangent vectors to $M^{k}$ at $p$. Then $H_{p}\left(M^{k}\right)$ is the maximal complex subspace of $T_{p}\left(C^{n}\right)$ which is contained in $T_{p}\left(M^{k}\right)$. It is well known that

$$
\max (k-n, 0) \leqq \operatorname{dim}_{C} H_{p}\left(M^{k}\right) \leqq[k / 2]
$$

Presented to the Society, March 1, 1971; received by the editors March 17, 1971 and, in revised form, August 2, 1971.

AMS 1969 subject classifications. Primary 3220, 3225; Secondary 3227.

Key words and phrases. Extendibility of holomorphic functions, C-R extendibility, the Levi form.

Copyright (C) 1972, American Mathematical Society 
There is another way of examining almost complex structures which we shall use. Let $f$ denote the embedding of $M^{k}$ into $C^{n}$, and let $J(f)$ be the complex Jacobian of $f$. If $q=\min (n, k)$, a point $p$ in $M^{k}$ is said to be an exceptional point of order $l$, $0 \leqq l \leqq[k / 2]-\max (k-n, 0)$ if the complex rank of $\left.J(f)\right|_{p}$ is equal to $q-l$.

A point $p$ in $M^{k}$ is generic if $p$ is an exceptional point of order 0 . The manifold $M^{k}$ is locally generic at $p$ if every point in some open neighborhood of $p$ is generic, and is locally $C-R$ at $p$ if every point in some open neighborhood of $p$ is an exceptional point of the same order.

Suppose $M^{k}$ is locally C-R at $p$ and $H_{p}\left(M^{k}\right)$ is nonempty. Then we define the Levi form at any $x$ near $p$

$$
L_{x}\left(M^{k}\right): H_{x}\left(M^{k}\right) \rightarrow\left(T x\left(M^{k}\right) \otimes C\right) /\left(H_{x}\left(M^{k}\right) \otimes C\right)
$$

by $L_{x}\left(M^{k}\right)(t)=\pi_{x}\left\{[Y, \bar{Y}]_{x}\right\}$, where $Y$ is a local section of the fiber bundle $H\left(M^{k}\right)$ (with fiber $H_{x}\left(M^{k}\right)$ ) such that $Y_{x}=t,[Y, \bar{Y}]_{x}$ is the Lie bracket evaluated at $x$, and

$$
\pi_{x}: T_{x}\left(M^{k}\right) \otimes C \rightarrow\left(T_{x}\left(M^{k}\right) \otimes C\right) /\left(H_{x}\left(M^{k}\right) \otimes C\right)
$$

is the projection.

Denote by $\mathcal{O}_{C^{n}}=\mathcal{O}$ the sheaf of germs of holomorphic functions on $\boldsymbol{C}^{n}$. Let $K$ be a compact subset of $C^{n}$ and $V$ an open subset of $C^{n}$ containing $K$. We set

$$
\mathcal{O}(K)=\underset{V \supset K}{\operatorname{ind} \lim } \mathcal{O}(V)
$$

where $\mathcal{O}(V)$ is the Fréchet algebra of holomorphic functions on $V$. We say that $K$ is extendible to a connected set $K^{\prime} \supsetneq K$ if the map $r: \mathcal{O}\left(K^{\prime}\right) \rightarrow \mathcal{O}(K)$ is onto.

Suppose $f \in \mathscr{C}^{\infty}\left(M^{k}\right)$. We say $f$ is a $C$ - $R$ function at $p \in M^{k}$ if $\bar{X} f(y)=0$, for $y$ near $p$ and $X$ any section of $H\left(M^{k}\right)$. If $M^{k}$ is locally C-R at $p$ it suffices to verify the equality just for $X$ in a local basis for $H\left(M^{k}\right)$ at $p$. We note that our manifold need not be globally C-R. Thus we may have points which are not locally C-R. But obviously, the set of such points is nowhere dense in $M^{k}$.

Definition 2.1. Let $f \in \mathscr{C}^{\infty}\left(M^{k}\right)$. Then $f$ is a $C$ - $R$ function on $M^{k}$ if $f$ is a C-R function at each point of $M^{k}$. The C-R functions are denoted by $\operatorname{CR}\left(M^{k}\right)$.

We say that $M^{k}$ is $C-R$ extendible to a connected set $K=M^{k} \cup K^{\prime}$, where $K^{\prime} \neq \varnothing$, if for every $f \in \mathrm{CR}\left(M^{k}\right)$ there exists an $F: M^{k} \cup K^{\prime} \rightarrow C$ continuous so that $\left.F\right|_{M^{k}}=f$ and $\left.F\right|_{K^{\prime}} \in \mathcal{O}\left(K^{\prime}\right)$. We observe that C-R extendibility implies extendibility.

Let $K$ be a compact subset of $C^{n}$. We shall call a point $x \in K$ a holomorphic peak point if there exists a function $f \in \mathcal{O}(K)$ such that, for any $y \in K-\{x\}$, we have $|f(y)|<|f(x)|$. 
3. Local equations and the Levi form. Again let $M^{k}$ be a real $k$-dimensional $\mathscr{C}^{\infty}$ manifold embedded in $C^{n}, k, n \geqq 2$. Suppose $M^{k}$ is locally C-R at $p$, and $p$ is an exceptional point of order $l$. If $k>n$ the local equations of $M^{k}$ in a neighborhood of $p$ are (after a suitable choice of coordinates)

(1)

$$
\begin{aligned}
& z_{1}=x_{1}+i h_{1}\left(x_{1}, \ldots, x_{2(n-l)-k}, w_{1}, \ldots, w_{k-n+l}\right) \\
& \vdots \\
& z_{2(n-l)-k}=x_{2(n-l)-k}+i h_{2(n-l)-k}\left(x_{1}, \ldots, x_{2(n-l)-k}, w_{1}, \ldots, w_{k-n+l}\right) \\
& z_{2(n-l)-k+1}=u_{1}+i v_{1}=w_{1} \\
& \vdots \\
& z_{n-l}=u_{k-n+l}+i v_{k-n+l}=w_{k-n+l} \\
& z_{n-l+1}=g_{1}\left(x_{1}, \ldots, x_{2(n-l)-k}, w_{1}, \ldots, w_{k-n+l}\right) \\
& \vdots \\
& z_{n}=g_{l}\left(x_{1}, \ldots, x_{2(n-l)-k}, w_{1}, \ldots, w_{k-n+l}\right),
\end{aligned}
$$

where $x_{1}, \ldots, x_{2(n-l)-k}, u_{1}, v_{1}, \ldots, u_{k-n+l}, v_{k-n+l}$ are local coordinates for $M^{k}$ in a neighborhood of $p$ vanishing at $p$, and $z_{1}, \ldots, z_{n}$ are coordinates for $\boldsymbol{C}^{n}$ vanishing at $p$. The real-valued functions $h_{1}, \ldots, h_{2(n-l)-k}$ as well as the complexvalued functions $g_{1}, \ldots, g_{l}$ vanish to order 2 at $p$. Because $M^{k}$ is locally C-R at $p$, the functions $g_{1}, \ldots, g_{l}$ must be complex-analytic functions of $w_{1}, \ldots, w_{k-n+l}$ (see [3]).

Letting $g_{j}=g_{j}^{\prime}+i g_{j}^{\prime \prime}, j=1, \ldots, l$, we find from [5] that the Levi form vanishes at $p$ if and only if the complex Hessians at $p$ of each of the functions $h_{1}, \ldots, h_{2(n-l)-k}$, $g_{1}^{\prime}, g_{1}^{\prime \prime}, \ldots, g_{l}^{\prime}, g_{l}^{\prime \prime}$ with respect to the variables $w_{1}, \ldots, w_{k-n+l}$ all have zero eigenvalues.

Fix $x_{1}, \ldots, x_{2(n-l)-k}$ and expand each $g_{j}$ in a Taylor series in $w_{1}, \ldots, w_{k-n+l}$,

$$
g_{j}=\sum_{\alpha} a_{j, \alpha} w^{\alpha},
$$

where $w=\left(w_{1}, \ldots, w_{k-n+l}\right)$ and $\alpha=\left(\alpha_{1}, \ldots, \alpha_{k-n+l}\right)$. Replacing $z_{n-l+j}$ by $z_{n-l+j}$ $-\sum_{\alpha} a_{j, \alpha} w^{\alpha}$, we have that $z_{n-l+1}=0, \ldots, z_{n}=0$ in our new local equations. Thus the Levi form vanishes at $p$ if and only if the complex Hessians at $p$ of each of the functions $h_{1}, \ldots, h_{2(n-l)-k}$ are all zero matrices.

Suppose $M^{k}$ is compact in $C^{n}$. It is shown in [5] that there exists an open set of holomorphic peak points on $M^{k}$ which is nonempty. By the remarks before Definition 2.1, we can find a holomorphic peak point $p \in M^{k}$ such that $p$ is an exceptional point of some order $l$, and $M^{k}$ is locally C-R at $p$. Assume $p=0$ and $M^{k}$ near $p$ is given by the equations in (1). Wells proves that through $p$ we can put a hyperplane which intersects $M^{k}$ at only the point $p$. If $z_{j}=x_{j}+i y_{j}, j=1, \ldots$, $2(n-l)-k, n-l+1, \ldots, n$, we can assume the hyperplane is defined by $y_{1}=0$ (the information about the $g_{j}$ 's in this section forces our arbitrary choice to $\left.y_{1}, \ldots, y_{2(n-l)-k}\right)$.

Let $Q$ denote the 1-dimensional real subspace of $T_{0}\left(C^{n}\right)$ generated by $\partial / \partial y_{1}$. Set 
$W=Q \oplus T_{0}\left(M^{k}\right)$ and let $\pi$ be the projection from $C^{n}$ to $W$. Under this projection the manifold $M^{k}$ projects to a manifold with local equations

$$
\begin{aligned}
& z_{1}=x_{1}+i h_{1}\left(x_{1}, \ldots, x_{2(n-l)-k}, w_{1}, \ldots, w_{k-n+l}\right) \\
& z_{2}=x_{2} \\
& \vdots \\
& z_{2(n-l)-k}=x_{2(n-l)-k} \\
& z_{2(n-l)-k+1}=u_{1}+i v_{1}=w_{1} \\
& \vdots \\
& z_{n-l}=u_{k-n+l}+i v_{k-n+l}=w_{k-n+l} .
\end{aligned}
$$

Wells shows that

$$
\frac{\partial^{2} h_{1}}{\partial x_{1}^{2}}, \ldots, \frac{\partial^{2} h_{1}}{\partial x_{2(n-l)-k}^{2}}, \frac{\partial^{2} h_{1}}{\partial u_{1}^{2}}, \ldots, \frac{\partial^{2} h_{1}}{\partial u_{k-n+l}^{2}}, \frac{\partial^{2} h_{1}}{\partial v_{1}^{2}}, \ldots, \frac{\partial^{2} h_{1}}{\partial v_{k-n+l}^{2}}
$$

are all $>0$ on some open neighborhood $U$ of $p$ in $M^{k}$. In particular

$$
\frac{\partial^{2} h_{1}}{\partial w_{1} \partial \bar{w}_{1}}, \ldots, \frac{\partial^{2} h_{1}}{\partial w_{k-n+l} \partial \bar{w}_{k-n+l}}
$$

are positive on the set $U$. By diagonalizing, we find that the Hessian of $h_{1}$ with respect to $w_{1}, \ldots, w_{k-n+l}$ is positive definite.

4. The main result. Assume $M^{k}$ is a real $k$-dimensional $\mathscr{C}^{\infty}$ manifold embedded in $C^{n}$, and $M^{k}$ is locally C-R at $p \in M^{k}$. Suppose at least one of the following conditions is satisfied.

(I) There is a real hypersurface containing $M^{k}$ whose Levi form restricted to $H\left(M^{k}\right)$ has at $p$ at least one positive and one negative eigenvalue.

(II) There is a real hypersurface containing $M^{k}$ whose Levi form restricted to $H\left(M^{k}\right)$ has at $p$ all its eigenvalues of the same sign different from zero.

Then we have the following theorem due to Nirenberg [4].

THEOREM 4.1. Let $M^{k}$ be locally $C-R$ at $p \in M$ and assume either (I) or (II) holds. Then $M^{k}$ is locally $C-R$ extendible to a manifold $\tilde{M}$ of real dimension one higher than that of $M^{k}$.

We are now able to prove the main result.

THEOREM 4.2. Let $M^{k}$ be a real $k$-dimensional compact $\mathscr{C}^{\infty}$ manifold embedded in $C^{n}, k>n \geqq 2$. Then $M^{k}$ is $C$ - $R$ extendible to a real $(k+1)$-dimensional submanifold of $C^{n}$.

Proof. We showed in the previous section that there exists a point $p \in M^{k}$ such that:

(i) $M^{k}$ is locally C-R at $p$,

(ii) $M^{k}$ is given by the local equations (1) near $p$, and

(iii) the complex Hessian of the function $h_{1}$ with respect to the variables $w_{1}$, $\ldots, w_{k-n+l}$ has all positive eigenvalues at $p$. 
Consider the real hypersurface containing $M^{k}$ defined by the function $\rho=y_{1}-h_{1}$. The Levi form of this hypersurface restricted to $H\left(M^{k}\right)$ is the negative of the complex Hessian of $h_{1}$ with respect to the variables $w_{1}, \ldots, w_{k-n+l}$. Then this hypersurface satisfies condition (II) at the point $p$, and we apply Theorem 4.1. Q.E.D.

THEOREM 4.3. Let $M^{k}$ be a real $k$-dimensional compact $\mathscr{C}^{\infty}$ manifold embedded in $C^{n}, k>n \geqq 2$. Then $M^{k}$ is extendible to a real $(k+1)$-dimensional submanifold of $\boldsymbol{C}^{n}$.

REMARK 1 . The manifold $\tilde{M}$ of Theorem 1 can be taken to have $\mathscr{C}^{q}$ structure, $1 \leqq q<\infty$.

REMARK 2 . If $k \leqq n$, then there are examples of totally real submanifolds which are always holomorphically convex. Thus, from the standpoint of dimension, Theorems 4.2 and 4.3 are the best possible.

\section{REFERENCES}

1. E. Bishop, Differentiable manifolds in complex Euclidean space, Duke Math. J. 32 (1965), 1-21. MR 34 \#369.

2. S. J. Greenfield, Cauchy-Riemann equations in several variables, Ann. Scuola Norm. Sup. Pisa (3) 22 (1968), 275-314. MR 38 \#6097.

3. L. R. Hunt, Real-analytic submanifolds of complex manifolds, Proc. Amer. Math. Soc. 29 (1971), 69-74.

4. R. Nirenberg, On the H. Lewy extension phenomenon (to appear).

5. R. O. Wells, Jr., Concerning the envelope of holomorphy of a compact differentiable submanifold of a complex manifold, Ann. Scuola Norm. Sup. Pisa (3) 23 (1969), 347-361. MR 39 \#7141.

6. - Holomorphic hulls and holomorphic convexity of differentiable submanifolds, Trans. Amer. Math. Soc. 132 (1968), 245-262. MR 36 \#5392.

Department of Mathematics, Texas Tech University, Lubbock, Texas 79409 